\title{
An Almost-Optimal Forward-Private RFID Mutual Authentication Protocol with Tag Control
}

\author{
Paolo D’Arco \\ Dipartimento di Informatica \\ Università degli Studi di Salerno \\ I-84084 Fisciano (SA), Italy
}

\begin{abstract}
In this paper we propose an efficient forward-private RFID mutual authentication protocol. The protocol is secure under standard assumptions. It builds over a recent work, extends it to achieve mutual authentication, and improves it by introducing a resynchronization mechanism between tag and reader, through which the server-side computation from $O(N \omega)$ is reduced to $O(N+\omega)$, where $N$ is the total number of tags in the system, and $\omega$ is the maximum number of authentications each single tag can afford during its lifetime. Moreover, the protocol enables the server to control how many times a tag has been read by legitimate and fake readers.
\end{abstract}

\section{Introduction}

Rfid Technology: basics, development and concerns. The Rfid technology enables automatic object identification without the need for physical access. Each object is labeled with a tiny integrated circuit equipped with a radio antenna, called tag, whose information content can be received by another device, called reader, at a distance of several meters. Usually the readers are connected to a back-end server: they forward to the server the read tag content, and get back the result of the server computation. The interest of the scientific community for the Rfid technology has grown a lot during the last years simultaneously to the wide diffusion of the technology and the deployment of applications which partially deal or embed Rfid components. Indeed, the indubitable advantages come with new challenges: security and privacy, due to the constrained computational capabilities of the tags, are non trivial properties to achieve. If some applications do not need stringent security and privacy measures, applications which have an impact on the people life style, raise more concerns: in some settings as users (e.g., in access control applications, in anti-theft tools) we would like to be sure that a certain tag cannot be impersonated by an adversary; as well as, there are uses in which tracking features (e.g., postal tracking, pet tracking, airline luggage tracking, waste disposal tracking) are very welcome but others (e.g., when buying tag-equipped goods from a shop) in which we would like to be sure that our privacy is preserved, and no adversary is able to build a preference profile by illegally reading the content of the tags attached to the goods we buy. 
State of art. We refer the interested reader to [1413] for an overview of the applications of the RFID technology and of the main security issues, and to [1] for references to research papers dealing with RFID technology and its challenges 1 .

Previous work. Roughly speaking, an Rfid authentication protocol enables tags and readers to be sure they are talking to each other, i.e., to identify and authenticate the other part. It is a key-component for building secure and private Rfid applications. An RFID authentication protocol is forward-private if an adversary, who tampers a tag and obtains its keys and state information, is unable to trace the tag, i.e., to associate the tag to previous transcripts of completed protocol executions he has eavesdropped. Obhuko et al. [15. proposed a simple and elegant forward-private scheme, which uses two hash functions. The scheme and its subsequent improvements, however, due to the costs of hash functions, are unsuitable for a real implementation on a tag. Moreover, such schemes are proven secure by using the random oracle methodology which is object of debate and criticism [26]. A recent paper [4] introduced a new Obhuko et al. like scheme, called PFP, which is efficient and is secure under standard assumptions, i.e., the existence of pseudorandom number generators and strongly universal hash function families.

Our contribution. In this paper we propose $E F P P$, a new forward-private RFID mutual authentication protocol. It builds over PFP and improves it by introducing a resynchronization mechanism between tag and reader, similar to the one used in [1112], through which the server-side computation from $O(N \omega)$ is reduced to $O(N+\omega)$, where $N$ is the total number of tags in the system, and $\omega$ is the maximum number of authentications each single tag can afford during its lifetime. Since the authors of [10, who focused on the design and the analysis of Rfid protocols based on symmetric-key primitives, showed that, if keys are chosen independently and uniformly at random, $\Omega(N)$ is a lower bound on the number of lookup operations the back-end server needs to authenticate a tag, then it follows that our forward-private scheme is almost optimal.

Related Work. Apart [4], which is our starting point, and [11]2], from which we borrow the resynchronization technique, other related papers are [17/5]. The OFRAP mutual authentication scheme, proposed in [17], is elegant, efficient, and forward-private. It has been analyzed within the UC framework and proven secure and private under standard assumptions, i.e., the existence of pseudorandom functions. Moreover, it achieves an $O(N)$ overhead in terms of the number of lookup operations the server needs to authenticate a tag. Compared to ours, apart the computational tools, the main difference is that in OFRAP the server has no way to control the total number of protocol executions a tag has been subject to, perhaps due to an adversary attack. In our scheme, on the other hand, we gain control by paying an additive $\omega$ factor within the asymptotic notation, which enables the back-end server to remove from the system a tag once its lifetime is over. On the other hand, the PEPS scheme [5] also

\footnotetext{
${ }^{1}$ In the full version of the paper 8 are briefly mentioned the most significant efforts in order to provide precise notions of security and privacy, and to propose efficient constructions.
} 
reduces the $O(N \omega)$ server-side computation of $P F P[4$ to $O(N)$. It has a design quite similar to $O F R A P$, and it is secure under the same assumptions. The main difference between PEPS and $O F R A P$ is that PEPS requires the tag to generate truly random numbers. We point out that also in $P E P S$ the server has no way to control the total number of protocol executions a tag has been subject to. In some applicative settings (e.g., access control, ticketing, automatic tolls ...) such a property is very welcome. The server could estimate the usage of the tag, as well as whether the tag has been target of attacks. EFPP is a true extension of $P F P$, which is efficient and practical, and uses the same computational tools. To our knowledge, EFPP is also the first efficient RFID forward-private authentication protocol enjoying the above tag-control feature.

\section{Security Model}

Every security model for evaluating Rfid authentication protocols focuses on three aspects: correctness, security and privacy. Loosely speaking, a protocol is correct if, with overwhelming probability, a legitimate tag and a legitimate reader successfully authenticate each other in an adversary-free protocol execution. Then, it is secure if an adversary has a negligible probability of impersonating a legitimate tag to the reader (vice versa, a reader to tag). Finally, it is private if a tag cannot be traced by analyzing the transcripts of protocol executions, and it is forward-private if the adversary does not succeed even if, at a certain point, gets access to the tag content and tries to trace the tag by using the transcript of previous completed executions.

Despite the properties we would like to get are intuitively clear, providing a suitable security and privacy model is a challenging task. Just to exemplify, the notion of correctness has to take into account a possible desynchronization attack tag and reader can be subject to at a certain point. What do we need to require from an adversary-free protocol execution after such an event has occurred? The models in 18/411] formalize this requirement in different ways. In this abstract, we do not deal with security model issues: since we basically use the same primitives of [4, for easiness of comparison, we refer to the same model (extended to deal with mutual authentication) which, as stated by the authors of [4, is a simplification and an adaptation of [1816] to the symmetric setting 2 .

The Model. Each tag $T$ has an internal state, containing state information and secret keys. Tag secret keys are uncorrelated, chosen independently and uniformly at random. Part of the tag state is shared with the back-end server, which stores tag information in a database DB. Each tag can be used at most $\omega$ times. Readers are securely connected to the back-end server. During its lifetime, a tag enters authentication exchanges with the readers, following a protocol which specifies which messages have to be computed and exchanged, and how the internal states of the tag and the back-end server have to be updated. An authentication exchange between a tag and a reader either results inside the reader (resp. tag)

\footnotetext{
${ }^{2}$ An analysis of the protocol in different models is left as future work.
} 
in an authentication success (together with a tag identity for the reader) or in an authentication failure. A tag cannot handle several authentication exchanges simultaneously. We assume that tags are exposed to an adversary during an exposure period, in which the adversary is able to observe and disturb all interactions involving the tag and possibly the reader, without confusing these interactions with exchanges involving other tags of the system. We also assume that no physical characteristics (e.g., radiation pattern, response time, et cetera) allow an adversary to recognize the tag and distinguish it from the other tags of the system, if the adversary observes it again in another exposure period.

Let $A$ be an adversary with running time upper-bounded by $T$, allowed to trigger, observe, disturb and replace up to $q<\omega$ authentication exchanges involving the tag and the reader, and to access the outcome of the authentication protocol. We say that $A$ is a $(q, T)$-adversary.

Definition 1. An Rfid authentication protocol is said to be $(q, T, \epsilon)$-correct iff the probability that a legitimate tag (resp. reader) is not successfully authenticated by a legitimate reader (resp. tag) in an undisturbed exchange at least once in its lifetime is upper-bounded by $\epsilon$, even in the presence of a $(q, T)$-adversary. The probability is taken over the initial tag's secret values, the random numbers chosen during the protocol executions, and the random numbers chosen by the adversary.

The definition states that a protocol is correct iff, even in presence of a $(q, T)$ adversary which tries to desynchronize tag and reader (i.e., so that they reach different states), the probability that in its lifetime there exists an adversary-free execution of the protocol in which the tag (resp. reader) is not authenticated by the reader (resp. tag), is at most $\epsilon$. In other words, the protocol is robust against a $(q, T)$-adversary and it works almost always well.

Security requires resistance to impersonation attacks, which can be modeled as two-stage processes: during the first stage a $(q, T)$-adversary interacts both with a legitimate reader and a legitimate tag. During the second stage, the adversary only interacts with the reader (resp. tag) and initiates an authentication exchange to impersonate the tag (resp. the reader). The attack succeeds if the authentication is successful and the adversary is identified as the tag (resp. as the reader).

Definition 2. An Rfid authentication protocol is said to be $(q, T, \epsilon)$-secure (w.r.t. tag authentication/w.r.t. reader authentication) iff, for any $(q, T)$-adversary, the probability that an impersonation attack is successful is at most $\epsilon$.

The privacy requirement can be formalized through the following privacy experiment: during the first stage, a $(q, T)$-adversary $A$ interacts with any two legitimate tags, $T_{0}$ and $T_{1}$, and a legitimate reader. At the end of this phase, a bit $b$ (concealed to $A$ ) is chosen. Then, during the second stage, $A$ again interacts with $T_{b}$. Then, $A$ is given access to the internal state of $T_{b}$. Eventually, $A$ outputs a guess bit $b^{\prime}$ for the value $b$, and it succeeds if $b^{\prime}$ is equal to $b$. 
Definition 3. An Rfid authentication protocol is said to be $(q, T, \epsilon)$-private iff any $(q, T)$-adversary $A$ has an advantage at most $\epsilon$ in winning the privacy experiment, i.e.,

$$
\mid \operatorname{Pr}[\text { A succeeds }]-1 / 2 \mid \leq \epsilon .
$$

Notice that, in the privacy experiment, we assume that $A$ is given access to the internal state of the tag when a protocol execution is completed. This precludes tag states following a failed protocol execution. The same approach was followed in [17] and, very recently, in [5], where the authors used the notion of almost forward private protocol to refer to the above setting with a restricted corruption capability for the adversary. However, we point out that, by using the same argument used in [16] (see Thm 1, page 294), it is possible to prove that if an adversary has the power to corrupt a tag during a protocol execution, then it easily wins the privacy experiment. Unfortunately, this issue has no protocol solution without extra hardware assumptions [169]. Therefore, the only possible goal is to look for a protocol which safely locks previous completed executions. 4 .

\section{Tools}

In this section we review some useful tools and properties needed to analyze the strength of the protocol. See [4] (Section 4 and the appendices) for proofs and details.

Let $L, n$ and $k$ be integers such that $L=n+k$, and let $g:\{0,1\}^{n} \rightarrow$ $\{0,1\}^{L}$ be a binary function, which expands $n$-bit sequences into $L$-bit sequences. A distinguisher for $g$ is a probabilistic algorithm $A$, which on input an $L$-bit sequence, outputs 0 or 1 . The advantage of $A$ in distinguishing $g$ from a perfect random generator is defined as:

$$
A d v_{g}(A)=|\operatorname{Pr}[A(g(x))=1]-\operatorname{Pr}[A(y)=1]|
$$

where the probabilities are taken over $x \in\{0,1\}^{n}$ (unknown to A) and $y \in$ $\{0,1\}^{L}$, chosen uniformly at random, and over the random bits chosen by $A$. The advantage in distinguishing $g$ in time $T$ is:

$$
A d v_{g}(T)=\max _{A}\left\{A d v_{g}(A)\right\}
$$

for all distinguishers $A$ running in time at most $T$.

Definition 4. The function $g:\{0,1\}^{n} \rightarrow\{0,1\}^{L}$ is a $(T, \epsilon)$-secure pseudorandom number generator $\left((T, \epsilon)-P R N G\right.$, for short) iff $A d v_{g}(T) \leq \epsilon$.

\footnotetext{
${ }^{3}$ Using the language of [11, the tag is clean at the corruption time, i.e., an undisturbed protocol execution with the reader has been successfully completed and the tag is ready for a new protocol execution.

${ }^{4}$ Notice that similar constraints to get perfect forward-secrecy in the key-exchange setting were shown in [3] (see Remark 7). It is the same problem which appears in two different settings.
} 
By using $\lambda$ times the function $g$, we can define an iterated function $G_{\lambda}$. To this aim, let us denote $g(x)=g_{1}(x) \| g_{2}(x)$, where $g_{1}(x) \in\{0,1\}^{n}, g_{2}(x) \in\{0,1\}^{k}$, and $\|$ represents concatenation. Then, let $\lambda$ be an integer greater than or equal to 1 . The iterated function $G_{\lambda}:\{0,1\}^{n} \rightarrow\{0,1\}^{n+\lambda k}$ is defined by:

$$
x \rightarrow\left(g_{2}(x), g_{2}\left(g_{1}(x)\right), \ldots, g_{2}\left(g_{1}^{\lambda-1}(x)\right), g_{1}^{\lambda}(x)\right) .
$$

Assuming that $T_{g}$ is the time to compute $g$, it holds that:

Theorem 1. If $g:\{0,1\}^{n} \rightarrow\{0,1\}^{n+k}$ is a $\left(T, \epsilon_{g}\right)-P R N G$ then, for any $\lambda \geq 1$, the associated iterated function $G_{\lambda}$ is a $\left(T-(\lambda+1) T_{g}, \lambda \epsilon_{g}\right)-P R N G$.

Similarly, a duplicated function $G^{N}:\{0,1\}^{n N} \rightarrow\{0,1\}^{L N}$ is simply defined by $\left(x_{1}, \ldots, x_{N}\right) \rightarrow\left(G\left(x_{1}\right), \ldots, G\left(x_{N}\right)\right)$. It holds that:

Lemma 1. If $G$ is a $\left(T^{\prime}, \epsilon_{G}\right)-P R N G$, then $G^{N}$ is a $\left(T^{\prime}, N \epsilon_{G}\right)-P R N G$.

Finally, a duplicated iterated function $G_{\lambda}^{N}:\{0,1\}^{n N} \rightarrow\{0,1\}^{(n+\lambda k) N}$ is defined by $\left(x_{1}, \ldots, x_{N}\right) \rightarrow\left(G_{\lambda}\left(x_{1}\right), \ldots, G_{\lambda}\left(x_{N}\right)\right)$.

Theorem 1 and Lemma 1 were proven in 4 by using standard hybrid arguments. From them, it follows that:

Theorem 2. For any $\left(T, \epsilon_{g}\right)-P R N G g:\{0,1\}^{n} \rightarrow\{0,1\}^{n+k}$, any $\lambda \geq 1$, and any $N \geq 1$, the associated duplicated function $G_{\lambda}^{N}:\{0,1\}^{n N} \rightarrow\{0,1\}^{(n+\lambda k) N}$ is $a\left(T-(\lambda+1) T_{g}, N \lambda \epsilon_{g}\right)-P R N G$.

The second key-tool we need in our construction are function families with special uniformity properties, referred to as universal classes of hash functions 7 ]. The idea of a universal class of hash functions is to define a collection $\mathcal{H}$ of hash functions in such a way that a random choice of a function $h \in \mathcal{H}$ yields a low probability that any two distinct inputs $x$ and $y$ will collide when their hashed values are computed using the function $h$. A more structured function family is defined as follows:

Definition 5. A family $\mathcal{H}=\left\{h_{s}:\{0,1\}^{\ell} \rightarrow\{0,1\}^{m}\right\}$ of hash functions is called $\epsilon$-almost strongly universal if and only if: $\forall a \in\{0,1\}^{\ell}, \forall b \in\{0,1\}^{m}$, it holds that $P r_{s \in S}\left[h_{s}(a)=b\right]=2^{-m}$, and $\forall a_{1} \neq a_{2} \in\{0,1\}^{\ell}, \forall b_{1}, b_{2} \in\{0,1\}^{m}$, it holds that $\operatorname{Pr}_{s \in S}\left[h_{s}\left(a_{2}\right)=b_{2} \mid h_{s}\left(a_{1}\right)=b_{1}\right] \leq \epsilon$.

Notice that, the first condition states that any input $a$ is mapped to any hashed value $b$ with probability $\frac{1}{2^{m}}$. The second states that, given that $a_{1}$ is mapped to $b_{1}$, the conditional probability that $a_{2}$ is mapped to $b_{2}$, for any $a_{2} \neq a_{1}$, is at most $\epsilon$. A $2^{-m}$-almost strongly universal hash function family $\mathcal{H}$ is called a strongly universal hash function family. Further details and applications can be found in 2019.

The following lemma was proven in 44. It states that an adversary who knows a pair $\left(a_{0}, h_{s}\left(a_{0}\right)\right)$ and a bunch of pairs $\left(a_{j}, b_{j}\right)$, with $b_{j} \neq h_{s}\left(a_{j}\right)$, has a small probability of guessing the correct value of the function $h_{s}(a)$ on a new randomly chosen value $a$. 
Lemma 2. Let $\mathcal{H}=\left\{h_{s}:\{0,1\}^{\ell} \rightarrow\{0,1\}^{m}\right\}$ be an $\epsilon$-almost strongly universal hash function family, let $s^{*}$ be a (secret) value randomly chosen in $S$, and let $A$ be a computationally unbounded adversary who tries to predict the value of $h_{s^{*}}$ on a randomly chosen input value $a$. Suppose that $A$ is given at most one pair $\left(a_{0}, b_{0}\right)$ and at most $p \leq \frac{1}{2 \epsilon}$ pairs $\left(a_{j}, b_{j}\right)$ such that $h_{s^{*}}\left(a_{0}\right)=b_{0}$ and, for $0<$ $j \leq p$, it holds that $h_{s^{*}}\left(a_{j}\right) \neq b_{j}$. Then,

$$
\operatorname{Pr}_{a \in\{0,1\}^{\ell}, s^{*}}\left[A(a)=h_{s^{*}}(a)\right]=2^{-\ell}+\epsilon(1+2 p \epsilon) .
$$

Lemma 3. If $s, s^{\prime}$ are chosen independently, then $\operatorname{Pr}_{s, s^{\prime} \in S}\left[h_{s}(a)=h_{s^{\prime}}(a)\right]=2^{-m}$.

In the following, we will denote with $T_{h}$ the time to compute the function $h_{s}$.

\section{Protocol Description}

In this section we introduce our protocol. Let us briefly describe $P F P$, the forward-private protocol proposed in [4] we start from. Let $g:\{0,1\}^{n} \rightarrow\{0,1\}^{n+k}$ be a PRNG, and let $\mathcal{H}=\left\{h_{s}:\{0,1\}^{\ell} \rightarrow\{0,1\}^{m}\right\}$ be a strongly universal hash function family. Moreover, let $g(x)=g_{1}(x) \| g_{2}(x)$, where $g_{1}(x) \in\{0,1\}^{n}$ and $g_{2}(x) \in\{0,1\}^{k}$. Each tag can be used at most $\omega$ times. It stores the description of $g$ and $\mathcal{H}$, and a state variable $\sigma$. The back-end server stores the same information for all tags in its database. The protocol works as follows:

1. The reader chooses uniformly at random a challenge $c \in\{0,1\}^{n}$ and sends $c$ to the tag.

2. The tag, receiving $c$, updates its state $\sigma$ and chooses a random function $h_{s}$ from $\mathcal{H}$ by computing $(\sigma, s)=\left(g_{1}(\sigma), g_{2}(\sigma)\right)$. Then, it computes $r=h_{s}(c)$, and sends $r$ to the reader.

3. The reader, for each $\operatorname{tag} T$, fetches into the database $D B$ the last known state for $\operatorname{tag} T$, say $\sigma_{j}^{T}$, and checks whether there exists an index $i \geq 0$ such that $j+i<\omega$ and $h_{g_{2}\left(g_{1}^{i}\left(\sigma_{j}^{T}\right)\right)}(c)=r$. If such an index is found, then the tag is authenticated. Otherwise, it is refused.

In other words, at each protocol execution, the reader checks in $D B$ along chains of at most $\omega$ elements if a match is found. The protocol is correct, secure and forward-private and, in a system with $N$ tags, it has complexity $O(N \omega)$. In the following we show how to improve the scheme in order to get mutual authentication and to reduce the complexity from $O(N \omega)$ to $O(N+\omega)$.

Let us start by describing the information held by tags and readers in the new protocol.

Common public information: two $d$-bit values $\mathrm{pad}_{1}, \mathrm{pad}_{2}$, used for padding, and the descriptions of a pseudorandom number generator $g$ (PRNG) and of a strongly universal hash function family $H$ (SUHF, for short). The PRNG $g$ is used for identification purposes, for updating tag information, and within the authentication process. The SUHF $H$ is used within the authentication process. 
As before, we split the values of $g:\{0,1\}^{n} \rightarrow\{0,1\}^{n+k}$ in two parts, i.e., $g(x)=g_{1}(x) \| g_{2}(x)$.

Tag information: a $(n-d)$-bit randomizer $C R$, an identification key $k$, a state variable $\sigma$, the two $d$-bit values $p a d_{1}$ and $\operatorname{pad}_{2}$, and the descriptions of $g$ and $H$

Reader information: a DB which stores the description of $g$ and $H$, the two values $\operatorname{pad}_{1}$ and $\mathrm{pad}_{2}$, and, for each tag, the tag identifier $I D$, a counter $C N T_{I D}$, and two tuples: $<I_{\text {old }}, \sigma_{\text {old }}, k_{\text {old }}, C R_{D B}^{\text {old }}>$ and $<I, \sigma, k, C R_{D B}>$. Let us denote by $D B_{I D}[i]$, for $i=0,1$, the memory locations for the two tuples for tag $I D$. At the beginning, when DB is initialized, all counters and old tuples are set to zero, i.e., $C N T_{I D}=0$ and $D B_{I D}[0]=<0,0,0,0>$ for all $N$ tags. The DB automatically removes tag $I D$ when $C N T_{I D}=\omega$. Moreover, let us denote by $\sigma_{\mid n-d}$ the first $n-d$ bits of $\sigma$, and by rnd a value chosen uniformly at random.

Three-round authentication protocol overview:

1. The reader chooses uniformly at random a challenge $c \in\{0,1\}^{n}$ and sends $c$ to the tag.

2. The tag updates its state, and computes and sends to the reader a triple, $\left(I, v_{T}, a u t h\right)$. The first two entries are used for identification and (if the synchronization is lost) to resynchronize tag and reader. More precisely, the value $I$ can be seen as a sort of pseudonym, which changes at each invocation, while the value $v_{T}$ contains information about the current randomizer $\mathrm{CR}$ of the tag. Finally, the value auth is the authenticator, used to authenticate the tag to the reader.

3. The reader, once received $\left(I, v_{T}, a u t h\right)$, looks in DB for a tuple starting with pseudonym $I$. If a tuple is found, the reader checks the received values are computed correctly from the tag, overwrites the old tuple for the tag with the current tuple, updates the current tuple $\left\langle I, \sigma, k, C R_{D B}\right\rangle$, and sends to the tag a value which acknowledges the received triple and authenticates the reader to the tag. Otherwise, it first tries to resynchronize with the tag and, then, it does the same check and update. If fails then it sends a random value to the tag.

4. The tag checks whether the received value is equal to the value it is expecting to receive and, accordingly, updates its key and outputs 1 or outputs 0 .

A complete description of the protocol, referred to as EFPP, is given in Figure1. The subroutines tag and reader invoke are described below.

\begin{tabular}{|l|l|}
\hline Compute $(c)$ & $\operatorname{Verify}\left(I D, c, I, v_{T}\right.$, auth $)$ \\
$I=g_{1}\left(\left(C R \| p a d_{1}\right) \oplus k\right)$ & $s=g_{2}(\sigma)$ \\
$\left(v_{0}, v_{1}\right)=g((c \oplus I) \oplus k)$ & if auth $\neq h_{s}\left(c \oplus I \oplus v_{T}\right)$ then return $(r n d, 0)$ \\
$v_{T}=\left(C R \| p a d_{2}\right) \oplus v_{0}$ & $\left(v_{0}, v_{1}\right)=g((c \oplus I) \oplus k)$ \\
$C R=C R+\sigma_{\mid n-d}$ & if $\left(C R_{D B} \| p a d_{2}\right) \neq v_{T} \oplus v_{0}$ then return $(r n d, 0)$ \\
$(\sigma, s)=\left(g_{1}(\sigma), g_{2}(\sigma)\right)$ & return $\left(v_{1}, 1\right)$ \\
auth $=h_{s}\left(c \oplus I \oplus v_{T}\right)$ & \\
return $\left(v_{1}, I, v_{T}\right.$, auth $)$ & \\
\hline
\end{tabular}




\section{Protocol steps:}

1. Reader: chooses $c \in\{0,1\}^{n}$ uniformly at random, and sends $c$ to the Tag.

2. Tag: sets $\left(v_{1}, I, v_{T}, a u t h\right)=\operatorname{Compute}(c)$ and sends $\left(I, v_{T}\right.$, auth $)$ to the Reader.

3. Reader: if there exists a tuple $t p=\left(I, \sigma, k, C R_{D B}\right)$ in DB for tag ID

(a) computes $\left(v_{1}, b\right)=\operatorname{Verify}\left(I D, c, I, v_{T}, a u t h\right)$

(b) if $(b==1)$ then invokes Update $(I D, t p)$ and outputs 1 ; else outputs 0

(c) sets $v_{R}=v_{1}$ and sends $v_{R}$

else, if no such a tuple exists, then

(a) sets $\left(C R_{N}, I D\right)=$ Lookupkey $\left(I, c, v_{T}\right)$ and $d=\operatorname{Resynch}\left(C R_{N}, I D\right)$

(b) if $(d==0)$ then sets $v_{1}=r n d$;

else $\left(v_{1}, b\right)=\operatorname{Verify}\left(I D, c, I, v_{T}, a u t h\right)$

if $(b==1)$ then Update $(I D, I D[1])$ and outputs 1 ; else outputs 0

(c) sets $v_{R}=v_{1}$ and sends $v_{R}$

4. Tag: if $v_{R}==v_{1}$, then sets $k=g_{1}(k)$ and outputs 1; else outputs 0

Fig. 1. EFPP: Efficient Forward-Private Protocol

\begin{tabular}{|l|}
\hline Update $(I D, t p)$ \\
$C N T_{I D}=C N T_{I D}+1$ \\
$D B_{I D}[0]=t p$ \\
$C R_{D B}=C R_{D B}+\sigma_{\mid n-d}$ \\
$\sigma=g_{1}(\sigma)$ \\
$k=g_{1}(k)$ \\
$I=g_{1}\left(\left(C R_{D B}|| p a d_{1}\right) \oplus k\right)$
\end{tabular}

\begin{tabular}{|c|c|}
\hline $\begin{array}{l}\text { Lookupkey }\left(I, c, v_{T}\right) \\
\text { look in DB for a key } k \text { for which } \\
\left(v_{0}, v_{1}\right)=g((c \oplus I) \oplus k) \text { are such that } \\
v_{T} \oplus v_{0}=\left(C R_{N} \| p a d_{2}\right) \\
\text { and } g_{1}\left(\left(C R_{N} \| p a d_{1}\right) \oplus k\right)=I \\
\text { if no } k \text { exists, then return }(0,0) \\
\text { else } D B_{I D}[1]=<I, \sigma, k, C R_{D B}> \\
\quad \operatorname{return}\left(C R_{N}, I D\right)\end{array}$ & $\begin{array}{l}\text { Resynch }\left(C R_{N}, I D\right) \\
\text { if }(0,0) \text { then return } 0 \\
\text { while }\left(C R_{D B} \neq C R_{N} \text { and } C N T_{I D}<\omega\right)\{ \\
\quad C R_{D B}=C R_{D B}+\sigma_{\mid n-d} \\
\quad \begin{array}{l}\sigma=g_{1}(\sigma) \\
\end{array} \quad C N T_{I D}=C N T_{I D}+1 \\
\} \\
\text { if } C N T_{I D}==\omega \text { then return } 0 \\
\quad \text { else return } 1\end{array}$ \\
\hline
\end{tabular}

\section{Properties}

The protocol enjoys several properties. Before going through formal proofs, and in order to get the ideas underlying the design, we provide some observations.

- Desynchronization attacks. An adversary might attack the system by sending multiple challenges $c$ to the tag. In such a way, the tag updates $C R$ and $\sigma$, which become different from $C R_{D B}$ and $\sigma$ stored in $D B$. However, notice 
that the identification key $k$, stays the same: it is updated only after a successful execution with the reader. In such a way, tag and reader, at the first adversary-free execution, recover the same value of $C R$ and resynchronize their states. Similarly, an adversary might discard the last message from the reader to the tag. Again, the attack fails since the server stores new and old tag records in $D B$.

- Computational efficiency. PFP, to authenticate the tag to the reader, requires $O(N \omega)$ iterations of the PRNG $g$. Our scheme requires $O(N+\omega)$ iterations of $g$. The small extra-amount of tag-side computation at each protocol execution consistently reduces the server-side computation.

- Forward-privacy. If an adversary corrupts the tag after a successful execution with the reader has occurred, he gets the current state and identification key. The assumptions on $g$ (i.e., it is a PRNG) guarantee the property 5 .

\section{Security Reductions}

In this section we show that the protocol is private, secure and correct.

Privacy. We prove that the privacy property holds by showing that if there exists an adversary $A_{p}$ which wins the privacy experiment for our protocol, then there exists an adversary $B_{p}$ which wins the privacy experiment for $P F P$ with the same advantage. Since $P F P$ is private, we conclude that our protocol is private, too. Essentially $B_{p}$ uses the context of his privacy experiment, to simulate the context of the privacy experiment for adversary $A_{p}$, which works against our protocol. Hence, we need to show how $B_{p}$ simulates the context for $A_{p}$ and why $A_{p}$ does not distinguish the simulated context from the real one. Adversary $B_{p}$ works as follows:

- $B_{p}$ starts the simulation of 2 'augmented' tags, $T_{0}^{\prime}$ and $T_{1}^{\prime}$, for the adversary $A_{p}$, by using the real tags $T_{0}$ and $T_{1}$ of the privacy experiment for $P F P$ he is interacting too.

- $B_{p}$ runs $A_{p} . B_{p}$ answers correctly all $A_{p}$ 's queries relaying modified queries to the tags and extending tags replies in phase 1 . The modified queries and replies are constructed as follows: if $A_{p}$ asks the reader to start a new execution, then $B_{p}$ chooses a uniformly at random $c$ and sends it to $A_{p}$. If $A_{p}$ sends $c$ to the tag, then $B_{p}$ chooses uniformly at random the values $I$ and $v_{T}$, and computes $c^{\prime}=c \oplus I \oplus v_{T}$. Then sends $c^{\prime}$ to the tag and gets back $h_{s}\left(c^{\prime}\right)$. Hence, constructs the triple $\left(I, v_{T}, h_{s}\left(c^{\prime}\right)\right)$, stores it in a database of simulated transcripts $S T D B$, and sends it to $A_{p}$. If $A_{p}$ sends the triple to the reader, then $B_{p}$ checks if the triple is in $S T D B$, simulates acceptance of the reader and sends (and stores in $S T D B$ ) $v_{1}$, chosen uniformly at random, to

\footnotetext{
${ }^{5}$ It is easy to see that, if an Adv corrupts the tag, for example, after sending it a challenge, then he gets the identification key $k$ (which stays the same as long as an adversary-free execution tag/reader does not occur) and can trace the tag by re-computing the pseudonym $I$. As we have stressed before, such corruptions during or after failed executions are precluded by the model.
} 
$A_{p}$. If $A_{p}$ sends $v_{1}$ to the tag, $B_{p}$ checks in $S T D B$ and simulates acceptance by the tag. Therefore, $B_{p}$ (using the real tags) is able to provide a partially simulated transcript to $A_{p}$.

- Let $b$ be the bit chosen by the privacy experiment runner $R$ for $P F P$, and let $T_{b}$ be the target tag. Since $B_{p}$ is simulating the privacy experiment for $A_{p}$, implicitly the choice of $R$ holds for $B_{p}$. (Let us assume that $R$ just removes from the scene $T_{b \oplus 1}$.) Then, $B_{p}$ keeps going with the simulation described before and, when $A_{p}$ asks to corrupt $T_{b}^{\prime}$, then $B_{p}$ corrupts $T_{b}$, and forwards to $A_{p}$ the state $\sigma$ (read in $T_{b}$ memory) and values chosen uniformly at random for $C R$ and $k$ to complete the amount of information which would be stored in a real tag $T_{b}^{\prime}$. Finally, $B_{p}$ outputs the same bit $b^{\prime}$ that $A_{p}$ outputs.

$B_{p}$ defeats the privacy of $P F P$ exactly with the same probability with which $A_{p}$ defeats the privacy of EFPP. What is left in the proof is to show that $A_{p}$ does not distinguish the simulated transcript from a real transcript.

To this aim, notice that the transcript of a protocol execution is given by tuples of the form $\left(c, I, v_{T}\right.$, auth, $\left.v_{1}\right)$, where $c$ is chosen uniformly at random, $I, v_{T}$ and $v_{1}$ are computed through the PRNG $g$, and auth is computed through the strongly universal hash function $h_{s}$, plus $C R$ and $k$, obtained by corrupting the tag after a successful protocol execution. Let $H_{0}, H_{1}, H_{2}$ and $H_{3}$ (hybrid) distributions of tuples defined as follows:

- $H_{0}$ contains tuples $\left(c, I, v_{T}, a u t h, v_{1}\right)$ computed like in the real protocol

- $H_{1}$ contains tuples $\left(c, I, v_{T}, a u t h, v_{1}\right)$ where $I$ is chosen uniformly at random

- $H_{2}$ contains tuples $\left(c, I, v_{T}, a u t h, v_{1}\right)$ where $I$ and $v_{T}$ are chosen uniformly at random

- $H_{3}$ contains tuples $\left(c, I, v_{T}, a u t h, v_{1}\right)$ where $I, v_{T}$ and $v_{1}$ are chosen uniformly at random

Notice that $H_{3}$ is the distribution of sequences produced in our simulation by $B_{p}$. By showing that, for $i=0,1,2$, it holds that $H_{i}$ is indistinguishable from $H_{i+1}$, we infer that $H_{0}$ is indistinguishable from $H_{3}$. To show that, for $i=0,1,2$, it holds that $H_{i}$ is indistinguishable from $H_{i+1}$, we use the same technique: if there exists a distinguisher $D_{H}$ between the two hybrids, then there exists a distinguisher $D_{g}$ which distinguishes the outputs of the PRNG from truly random values. Let us report the proof for the first case.

Let $H_{i}$ and $H_{j}$ be two distributions over sequences of $m$ tuples. We say that $H_{i}$ and $H_{j}$ are $(T, \epsilon)$-indistinguishable, iff $A d v_{D_{H_{i}, H_{j}}}(T) \leq \epsilon$, for any distinguisher $D_{H_{i}, H_{j}}$ running in time at most $T$.

Lemma 4. If $g$ is a $\left(T, \epsilon_{g}\right)$-secure $P R N G$, then $H_{0}$ and $H_{1}$ are $\left(T_{i}, \epsilon_{i}\right)$-indistinguishable where $T_{i}=T-3(m-1) T_{g}-(m-1) T_{h}$ and $\epsilon_{i}=\epsilon_{g} m^{2}$.

Proof. Let $H_{0,0}$ be a sequence of $m$ tuples, generated according to distribution $H_{0}$, and let $H_{0, m}$ be a sequence of $m$ tuples, generated according to distribution $H_{1}$. Moreover, for $i=1, \ldots, m-1$, let $H_{0, i}$ be a sequence of $m$ tuples, generated by choosing in the first $i$ tuples the value $I$ uniformly at random, and 
the remaining $m-i$ as in $H_{0}$. If there exists a $\left(T_{H}, \epsilon_{H}\right)$ distinguisher $D_{H_{0}, H_{1}}$ for the distributions $H_{0}$ and $H_{1}$, then there exists an index $i$ such that $D_{H_{0}, H_{1}}$ distinguishes between $H_{0, i}$ and $H_{0, i+1}$ with advantage $\geq \epsilon_{H} / m$. We construct a distinguisher $D_{g}$, by using $D_{H_{0}, H_{1}}$ as a subroutine, as follows:

- Let $V$ be the challenge-value $D_{g}$ has to decide (PRNG output or Random).

- Chooses uniformly at random an index $i \in\{1, \ldots, m-1\}$.

- Constructs a sequence of tuples $H_{c}$ by following the distribution $H_{0, i}$.

- Substitutes in the $i$-th tuple the value of $I$ with the challenge-value $V$ and provides $H_{c}$ to $D_{H_{0}, H_{1}}$.

- If $D_{H_{0}, H_{1}}$ outputs 0 , then $D_{g}$ outputs 0 . Else $D_{g}$ outputs 1 .

Notice that, when $V=g_{1}\left(\left(C R \| p a d_{1}\right) \oplus k\right)$ then $H_{c}=H_{0, i}$. On the other hand, when $V$ is a random value, then $H_{c}=H_{0, i+1}$. It follows that $D_{g}$ distinguishes the output of the PRNG from a random value with advantage $\geq \epsilon_{H} / m^{2}$. Moreover, $D_{g}$ has running time upper bounded by $T_{H}+3(m-1) T_{g}+(m-1) T_{h}$. Since by assumption $g$ is $\left(T, \epsilon_{g}\right)$-secure, if $T_{H}+3(m-1) T_{g}+(m-1) T_{h}<T$, we get that $\epsilon_{H} / m^{2} \leq \epsilon_{g}$, from which it follows that $H_{i}$ and $H_{j}$ are $\left(T_{i}, \epsilon_{i}\right)$-indistinguishable, where $T_{i}=T-3(m-1) T_{g}-(m-1) T_{h}$ and $\epsilon_{i} \leq \epsilon_{g} m^{2} . \triangle$

Similarly, we can show that if $A_{p}$ distinguishes $C R$ and $k$ of the simulated transcript from $C R$ and $k$ obtained by opening a tag after a real successful protocol execution, then we can construct a distinguisher for the PRNG $g$. It follows that $A_{p}$ does not distinguish a simulated transcript from a real one and we conclude that:

Theorem 3. If PFP is $\left(q, T, \epsilon_{p}\right)$-private, then EFPP is $\left(q, T, \epsilon_{p}\right)$-private.

Secure tag authentication. Notice that, restricting the attention to the first two rounds, our protocol generalises PFP. The first round is the same. In the second, the tag sends a triple (containing a value of $h_{s}$ ) instead of a single value $h_{s}$. Along the same line of the proof of [4], we show that, if there exists an efficient adversary $A$, which is able to impersonate a tag to the reader, then there exists an efficient distinguisher $B$ capable of distinguishing outputs of the PRNG $G_{\omega}$ from random values in $\{0,1\}^{\omega k}$. By suitably choosing the PRNG $g$, we show that the probability with whom $B$ (and hence $A$ ) succeeds is small. More precisely, we construct $B$ as follows:

$-B$ receives in input the sequence of values $z_{1}, \ldots, z_{\omega}$ it has to decide from which source it comes from.

- Then, it uses the above values $z_{1}, \ldots, z_{\omega}$ to simulate the computations of the tag $T$ (of unknown state) and the reader with whom $A$ is supposed to interact. More precisely, $B$ answers all $A$ 's queries in phase 1 as follows: if $A$ asks the reader to start a new execution, then $B$ chooses a uniformly at random $c$ and sends it to $A$. If $A$ sends $c$ to the tag then, assuming it is the $i$-th execution, $B$ chooses uniformly at random the values $I, v_{1}$ and $v_{T}$, sets $s=z_{i}$, and computes and sends to $A$ the triple $\left(I, v_{T}, h_{s}\left(c \oplus I \oplus v_{T}\right)\right)$. It also stores the triple and $v_{1}$ in $S T D B$. If $A$ sends the triple to the reader, then 
$B$ checks in $S T D B$ whether there exists an entry which matches the triple, simulates acceptance of the reader and sends $v_{1}$ to $A$. If $A$ sends $v_{1}$ to the tag, $B$ checks in $S T D B$ and simulates acceptance of the tag.

- Let $c$ be the challenge on which $A$, in phase 2, tries to impersonate $T$. $B$ gets back from $A$ the triple $\left(I, v_{T}, h_{s}\left(c \oplus I \oplus v_{T}\right)\right)$. $B$ checks whether $h_{s}\left(c \oplus I \oplus v_{T}\right)=h_{z_{q+1}}\left(c \oplus I \oplus v_{T}\right)$ ( $A$ has interacted $q<\omega$ times with tag and reader in phase 1 ) and, if the check is satisfied then accepts and outputs 1 ; otherwise, it outputs 0 .

Notice that, if $z_{1}, \ldots, z_{\omega}$ is pseudorandom (let us denote it as $Z_{G_{\omega}}$ ), then $B$ outputs 1 with probability $p_{A}$. Indeed, it is possible to show that the transcript of the simulated executions is indistinguishable from the transcript of real executions and, by assumption, on real transcripts, $A$ impersonates the tag with probability $p_{A}$. The indistinguishability can be shown by using standard arguments: if $A$ distinguishes between the transcripts, then can be constructed an efficient distinguisher for $g$. On the other hand, following the reasoning of [4] and applying Lemma 2, simple computations show that, if $z_{1}, \ldots, z_{\omega}$ are truly random values (let us denote them as $Z_{U}$ ), the probability that $B$ outputs 1 is less than $\omega\left(2^{-\ell}+\epsilon(1+2 q \epsilon)\right)$. It follows that:

$$
\left|\operatorname{Pr}\left[B\left(Z_{G_{\omega}}\right)=1\right]-\operatorname{Pr}\left[B\left(Z_{U}\right)=1\right]\right| \geq p_{A}-\omega\left(2^{-\ell}+\epsilon(1+2 q \epsilon)\right) .
$$

However, if $g$ is a $\left(T, \epsilon_{g}\right)$-secure PRNG, applying Theorem 11 we get that the advantage $\left|\operatorname{Pr}\left[B\left(Z_{G_{\omega}}\right)=1\right]-\operatorname{Pr}\left[B\left(Z_{U}\right)=1\right]\right| \leq \omega \epsilon_{g}$. The last two equalities show that $p_{A} \leq \omega\left(\epsilon_{g}+2^{-\ell}+\epsilon(1+2 q \epsilon)\right.$. Moreover, $B$ 's running time is equal to $A$ 's running time plus $q$ computations of $h_{s}$ for the tag simulation. Therefore, we can conclude that:

Theorem 4. If $\mathcal{H}$ is an $\epsilon$-almost strongly universal hash function family, $g$ is $a\left(T, \epsilon_{g}\right)$-secure PRNG, and $q \leq 1 / 2 \epsilon$, then EFPP is $\left(q, T^{\prime}, \epsilon_{s}\right)$-secure (w.r.t tag authentication) with $T^{\prime}=T-(\omega+1) T_{g}-q T_{h}$ and $\epsilon_{s}=\omega\left(\epsilon_{g}+2^{-\ell}+\epsilon(1+2 q \epsilon)\right)$.

Secure reader authentication. An adversary $A$, to be authenticated as reader from the $\operatorname{tag} T$, has to send in the third round of the protocol the right value $v_{1}$ to $T$. By using the same argument and simulation we have used before we show that, if there is an efficient $A$ who guesses $v_{1}$ with probability $p_{A}$, then there exists a distinguisher $B$ which distinguishes outputs of the PRNG $G_{\omega}$ from random values in $\{0,1\}^{\omega k}$, and then, by suitably choosing the PRNG $g$, we show that the probability $p_{A}$ is small. The distinguisher $B$ uses $A$ as a subroutine and simulates $A$ 's interaction with tag and reader. Eventually, if $A$ impersonates the reader, then $B$ outputs 1 . Otherwise, if $A$ fails, then $B$ outputs 0 . When the sequence $z_{1}, \ldots, z_{\omega}$ is chosen uniformly at random, then $B$ outputs 1 with probability at least $\frac{1}{2^{k}}$. On the other hand, if $z_{1}, \ldots, z_{\omega}$ is pseudorandom, then, $B$ outputs 1 with probability $p_{A}$. Indeed, as argued before, the simulated values received by $A$ from $B$ are indistinguishable from the values of real executions with the $\operatorname{tag} T$ and, by assumption, on real transcripts, $A$ impersonates the reader with probability $p_{A}$. It follows that:

$$
A d v_{G_{\omega}}(B)=\left|\operatorname{Pr}\left[B\left(Z_{G_{\omega}}\right)=1\right]-\operatorname{Pr}\left[B\left(Z_{U}\right)=1\right]\right| \geq p_{A}-1 / 2^{k}
$$


Due to Theorem[1, if $g$ is a $\left(T, \epsilon_{g}\right)$-secure PRNG, then it follows that $A d v_{G_{\omega}}(B) \leq$ $\omega \epsilon_{g}$. Hence, it holds that $p_{A} \leq 1 / 2^{k}+\omega \epsilon_{g}$. In conclusion:

Theorem 5. If $g$ is a $\left(T, \epsilon_{g}\right)$-secure PRNG, then EFPP is $\left(q, T^{\prime}, \omega \epsilon_{g}\right)$-secure (w.r.t. reader authentication) where $T^{\prime}=T-(\omega+1) T_{g}-q T_{h}$.

Correctness. In an adversary-free execution, a tag $T$ (resp. reader) is not authenticated by the reader (resp. tag), only if the reader updated twice the tuple associated to the tag in the database $D B$ and the tag did not or the tag updated its secret key $k$ and the reader did not. Such events happen only if

1. The adversary is able to impersonate the $\operatorname{tag} T$ (resp. the reader).

2. Collisions of $g$ and $h$ occur.

Due to the security of the scheme, as we have seen before, the first possibility happens with small probability. Hence, we do not need to care about it. Regarding the second, collisions of $g$ and $h$, we need to consider two separate cases: during an execution of the protocol, at a certain point, there exists a tuple in $D B$ associated to another tag either with the same $I$ and matching equations or with a different $I^{\prime}$ but a secret key $k$ by means of which we get a collision on $c, I, v_{T}$, and auth. More precisely, in the first case there exists in $D B$ a tuple $\left(I, k^{\prime}, \sigma^{\prime}, C R_{D B}^{\prime}\right)$, associated to tag $I D^{\prime} \neq I D$, for which $\left(v_{0}, v_{1}\right)=g\left(c \oplus I \oplus k^{\prime}\right)$ are such that:

$v_{T} \oplus v_{0}=C R_{D B}^{\prime} \| \operatorname{pad}_{2} \bigwedge g_{1}\left(\left(C R_{D B}^{\prime} \| p a d_{1}\right) \oplus k^{\prime}\right)=I \bigwedge h_{s^{\prime}}\left(c \oplus I \oplus v_{T}\right)=$ auth

while, in the second, there exists a tuple $\left(I^{\prime}, k^{\prime}, \sigma^{\prime}, C R_{D B}^{\prime}\right)$ for which $\left(v_{0}, v_{1}\right)=$ $g\left(c \oplus I \oplus k^{\prime}\right)$ are such that:

$$
v_{T} \oplus v_{0}=C R_{N} \| p a d_{2} \bigwedge g_{1}\left(\left(C R_{N} \| p a d_{1}\right) \oplus k^{\prime}\right)=I \bigwedge h_{s^{\prime}}\left(c \oplus I \oplus v_{T}\right)=\text { auth }
$$

Notice that $g_{1}\left(\left(C R_{D B}^{\prime} \| p a d_{1}\right) \oplus k^{\prime}\right)=I$ implies that $g_{1}\left(\left(C R_{D B} \| p a d_{1}\right) \oplus k\right)=$ $g_{1}\left(\left(C R_{D B}^{\prime} \| p a d_{1}\right) \oplus k^{\prime}\right)=I$ i.e., $g$ produces a collision. If $g$ is a $\left(T, \epsilon_{g}\right)$-secure PRNG, then it produces collisions with probability less than $\epsilon_{g}$. Otherwise, it would possible to construct a simple distinguisher for $g$ which distinguishes pseudorandom values from truly random values with probability higher than $\epsilon_{g}$. Moreover, due to Lemma 3, the equality $h_{s}\left(c \oplus I \oplus v_{T}\right)=h_{s^{\prime}}\left(c \oplus I \oplus v_{T}\right)=a u t h$, for $s \neq s^{\prime}$, occurs with probability $1 / 2^{m}$. A similar analysis applies to the second case. In conclusion, it holds that:

Lemma 5. If $\mathcal{H}$ is an $\epsilon$-almost strongly universal hash function family and $g$ is a $\left(T, \epsilon_{g}\right)$-secure PRNG, a collision during an execution of the protocol occurs with probability $<2 \cdot \epsilon_{g} / 2^{m}=\epsilon_{g} / 2^{m-1}$.

We need to consider the probability of collisions within the lifetime of the protocol. If the system has $N$ tags, since each tag can be used at most $\omega$ times, the protocol is useful for at most $N \omega$ authentications. By using the above result, we get that the probability of a collision within the system is $p_{c} \leq(N-1) \omega^{2} \epsilon_{g} / 2^{m-1}$. 
Let $g$ be a $\left(T, \epsilon_{g}\right)$-secure PRNG, where $T \geq(N-1) \omega^{2} T_{h}+(\omega+1) T_{g}$. Theorem 2 shows that the PRNG $G_{\omega}^{N}$, constructed from $g$ (which models tag state updates and the generation of seeds for $\left.h_{s},\right)$ is an $\left((N-1) \omega^{2} T_{h}, N \omega \epsilon_{g}\right)$-secure PRNG. Applying the same steps of 4 , we can conclude that the probability of failure of the protocol is $p<(N-1) \omega^{2} \epsilon_{g} / 2^{m-1}+N \omega \epsilon_{g}+\epsilon_{s}$, where $\epsilon_{s}$ is the probability of impersonation. In conclusion:

Theorem 6. Let g be a $\left(T, \epsilon_{g}\right)$-secure $P R N G$ where $T \geq(N-1) \omega^{2} T_{h}+(\omega+1) T_{g}$, let $\mathcal{H}$ be an $\epsilon$-almost strongly universal hash function family, and let $q \leq 1 / 2 \epsilon$. The EFPP authentication protocol is $\left(q, T^{\prime}, \epsilon_{c}\right)$-correct, with $T^{\prime}=T-(\omega+$ 1) $\left(3 T_{g}+q T_{h}\right)$ and $\epsilon_{c}=(N-1) \omega^{2} \epsilon_{g} / 2^{m-1}+N \omega \epsilon_{g}+\epsilon_{s}$.

\section{Conclusions}

We have proposed an efficient forward-private RFID mutual authentication protocol, secure under the assumption that exist secure pseudorandom number generators and strongly universal hash function families. At each authentication, compared to PFP where the tag computes one time the PRNG $g$ and one time the hash function $h_{s}$, the tag has to apply 3 times $g$ and one time $h_{s}$. On the other hand, the server, to authenticate a tag, in the worst case, instead of $O(N \omega)$ evaluations of $g$ and $h_{s}$ as in $P F P$, needs only $O(N+\omega)$ evaluations, where $N$ is the total number of tags in the system, and $\omega$ is the maximum number of authentications each single tag can afford during its lifetime. The server has full control over the number of protocol executions a tag has been subject to. The full version of this paper [8] reports an experimental comparison of $P F P$ vs $E F P P$, obtained by implementing the protocols.

\section{Acknowledgment}

The work described in this paper has been supported in part by the European Commission through the ICT program under contract 216676 ECRYPT II, in part by the Italian Ministry of University and Research - Project PRIN 2008 PEPPER: Privacy and Protection of Personal Data (prot. 2008SY2PH4), and in part by Project MTM2010-15167 from the Spanish Ministry of Science and Technology.

\section{References}

1. Avoine, G.: RFID Security and Privacy Lounge, http://www.avoine.net/rfid/

2. Bellare, M., Rogaway, P.: Random oracles are practical: A paradigm for designing efficient protocols. In: Proceedings of the First Annual Conference on Computer and Communications Security. ACM, New York (1993)

3. Bellare, M., Pointcheval, D., Rogaway, P.: Authenticated key exchange secure against dictionary attacks. In: Preneel, B. (ed.) EUROCRYPT 2000. LNCS, vol. 1807, pp. 139-155. Springer, Heidelberg (2000) 
4. Berbain, C., Billet, O., Etrong, J., Gilbert, H.: An Efficient Forward Private RFID Protocol. In: 16th ACM Conference on Computer and Communications Security (CCS 2009), pp. 43-53 (2009)

5. Billet, O., Etrog, J., Gilbert, H.: Lightweight Privacy Preserving Authentication for RFID Using a Stream Cipher. In: Hong, S., Iwata, T. (eds.) FSE 2010. LNCS, vol. 6147, pp. 55-74. Springer, Heidelberg (2010)

6. Canetti, R., Goldreich, O., Halevi, S.: The Random Oracle Methodology, Revisited. In: Proceedings of ACM STOC (1998)

7. Carter, J.L., Wegman, M.N.: Universal classes of hash functions. J. Computer and System Sci. 18, 143-154 (1979)

8. D'Arco, P.: An Almost-Optimal Forward-Private RFID Mutual Authentication Protocol with Tag Control, http://www.dia.unisa.it/paodar

9. D'Arco, P., Scafuro, A., Visconti, I.: Revisiting DoS Attacks and Privacy in RFIDEnabled Networks. In: 5th International Workshop on Algorithmic Aspects of Wireless Sensor Networks (ALGOSENSORS 2009). LNCS, vol. 5304, pp. 76-87 (2009)

10. Damgård, I., Østergaard, M.: RFID Security: Tradeoffs between Security and Efficiency. In: Proceedings of the RSA Conference, Cryptographers' Track, pp. 318-332 (2008)

11. Deng, R., Li, Y., Yao, A., Yung, M., Zhao, Y.: A New Framework for RFID Privacy, eprint archive, report no. 2010/059, http://eprint.iacr.org/

12. Deng, R., Li, Y., Yung, M., Zhao, Y.: A New Framework for RFID Privacy. In: Gritzalis, D., Preneel, B., Theoharidou, M. (eds.) ESORICS 2010. LNCS, vol. 6345, pp. 1-18. Springer, Heidelberg (2010)

13. Juels, A.: The Vision of Secure RFID. Proceedings of the IEEE 95(8), 1507-1508 (2007)

14. Juels, A., Pappu, R., Garfinkel, S.: RFID Privacy: An Overview of Problems and Proposed Solutions. IEEE Security and Privacy 3(3), 34-43 (2005)

15. Ohkubo, M., Suzuki, K., Kinoshita, S.: Efficient hash-chain based RFID privacy protection scheme. In: Proc. of the International Conference on Ubiquitous Computing Ubicomp Workshop Privacy: Current Status and Future Directions, Nottingham, England (September 2004)

16. Paise, R., Vaudenay, S.: Mutual Authentication in RFID: Security and Privacy. In: Proceedings of Aisaccs 2008, Lecture Notes in Computer Science, pp. 292-299 (2008)

17. Van Le, T., Burmester, M., de Medeiros, B.: Universally Composable and ForwardSecure Rfid Authentication and Authenticated Key Exchange. In: Proc. of ASIACCS 2007, pp. 242-252 (2007)

18. Vaudenay, S.: On Privacy Models for RFID. In: Kurosawa, K. (ed.) ASIACRYPT 2007. LNCS, vol. 4833, pp. 68-87. Springer, Heidelberg (2007)

19. Stinson, D.R.: Universal hashing and authentication codes. Designs, Codes and Cryptography (4), 369-380 (1994)

20. Wegman, M.N.: New hash functions and their use in authentication and set equality. Journal of Computer and System Sciences 22(3), 265-279 (1981) 